\title{
Variation in Coding Practices for Vestibular Schwannoma Surgery
}

\author{
Wenya Linda $\mathrm{Bi}^{1}$ Michael A. Mooney ${ }^{2}$ Seungwon Yoon ${ }^{2}$ Saksham Gupta ${ }^{1}$ Michael T. Lawton ${ }^{2}$ \\ Kaith K. Almefty ${ }^{2}$ C. Eduardo Corrales ${ }^{3}$ lan F. Dunn ${ }^{1}$ \\ ${ }^{1}$ Department of Neurosurgery, Center for Skull Base and Pituitary \\ Surgery, Brigham and Women's Hospital, Harvard Medical School, \\ Boston, Massachusetts, United States \\ 2 Department of Neurosurgery, Barrow Neurological Institute, St. Joseph's \\ Hospital and Medical Center, Phoenix, Arizona, United States \\ ${ }^{3}$ Division of Otolaryngology, Brigham and Women's Hospital, \\ Harvard Medical School, Boston, Massachusetts, United States \\ J Neurol Surg B 2019;80:96-102. \\ Address for correspondence Ian F. Dunn, MD, FAANS, FACS, \\ Department of Neurosurgery, Brigham and Women's Hospital, 60 \\ Fenwood Road, BTM 4, Boston, MA 02115, United States \\ (e-mail: idunn@partners.org).
}

\begin{abstract}
Keywords

- vestibular schwannoma

- acoustic neuroma

- skull base surgery

- CPT coding

- practice patterns
\end{abstract}

Introduction Nationwide databases are frequently used resources for assessing practice patterns and clinical outcomes. However, analyses based on billing codes may be limited by the inconsistent application of current procedural terminology (CPT) codes to specific operations. We investigated the variability among commonly used CPT codes for vestibular schwannomas resection and sought to identify factors that underlie this variation.

Methods The surgical procedure for 274 cases of vestibular schwannoma resections from two institutions was reviewed and classified as retrosigmoid, translabyrinthine, or middle fossa approaches. We then assessed the CPT codes assigned to each case and analyzed their association with surgical approach, surgeons involved, the coding specialty, and year of surgery. We further compared the incidence of CPT codes assigned for vestibular schwannoma surgeries in the American College Surgeons National Surgical Quality Improvement Program (NSQIP) database from 2010 to 2014. Results The majority (65\%) of vestibular schwannoma resections within the institutional cohort were billed with skull base approach and/or excision codes, whereas $76 \%$ of cases in NSQIP were associated with a single craniotomy for tumor code. The use of skull base codes over the past decade increased within our institutional cohort but remained relatively stable within NSQIP. CPT codes did not consistently reflect the operative approaches for vestibular schwannomas.

Conclusion We observed significant variability in coding patterns for vestibular schwannoma surgeries within institutions, surgical practices, and national databases. These results call for discretion in interpretation of data from aggregated billing codebased nationwide databases and suggests a role for institutional standardization of CPT assignments for the same approaches.

\section{Introduction}

Nationwide databases are powerful resources in analyses of outcomes for neurosurgical diseases. ${ }^{1}$ Their large sample sizes afford high statistical power to minimize the potential bias inherent to individual institutional data and provide valuable epidemiological data over time. Surgical cases in databases often contain one or more current procedural terminology (CPT) codes to describe the associated operation. CPT codes, in received

April 29, 2018

accepted

June 10, 2018

published online

July 16, 2018 (c) 2019 Georg Thieme Verlag KG Stuttgart · New York
DOI https://doi.org/ 10.1055/s-0038-1667124. ISSN 2193-6331. 
turn, offer an opportunity to trend practice patterns and compare outcomes between different approaches. Databases that include CPT coding are especially relevant for relatively uncommon pathologies such as vestibular schwannoma. ${ }^{2-8}$

Despite these advantages, increased attention has been directed toward the limitations and potential inaccuracies of national databases for neurosurgical research. ${ }^{9,10}$ While the inter-rater reliability of databases has been rated as good, there remains limited knowledge on whether databases precisely reflect the nuances of coding for skull base pathologies. ${ }^{11} \mathrm{CPT}$ coding practices may vary based on individual practice patterns, the integration of multidisciplinary teams, reimbursement associated with different combinations of CPT codes, and individual practice knowledge and literacy regarding code submission. Consequently, we investigated the variability among CPT codes for vestibular schwannoma resection and sought to identify factors that underlie this variation.

\section{Methods}

\section{Institutional Data Review}

Surgical resections of vestibular schwannoma performed at the Brigham and Women's Hospital between 2005 and 2017 and the Barrow Neurological Institute between 2013 and 2017 were retrospectively reviewed for histopathologic confirmation of diagnosis, operative approach, surgeon affiliation (neurosurgery, neurotology, or both), and CPT code. The surgical procedure for each case was classified as a retrosigmoid/ transmastoid retrosigmoid, translabyrinthine, or middle fossa approach. We then assessed the CPT codes assigned to each case and analyzed the variability in CPT codes based on surgical approach, primary surgeon, and year of procedure.

Cases assigned CPT codes 61518, 61520, and 61526 had a single primary code for craniotomy for tumor. For surgeries with skull base approach and resection CPT codes, 61590 to 61598 were considered approach codes, while CPT codes 61606 to 61616 were considered tumor excision codes. Ancillary codes for stereotactic image guidance (61781), use of operating microscope (69990), and others were considered separately. CPT codes were defined by the CPT-4 codebook (standard edition 2017), developed by the American Medical Association. (- Table 1).

\section{Nationwide Database Review}

We further assessed the incidence of primary and secondary CPT codes assigned for vestibular schwannoma surgeries in the American College Surgeons National Surgical Quality Improvement Program (ACS-NSQIP) annual databases from 2010 to -2014 . All NSQIP databases contain a primary CPT code and up to 20 secondary codes performed for each case. Duplicate codes were removed prior to analysis under presumption that they may have been erroneously inserted twice for the same case. NSQIP databases prior to 2010 were not included since secondary CPT codes were not routinely recorded until 2010.

Data contained within the multi-institutional NSQIP databases are collected prospectively by surgical reviewers on an 8day cycle to mitigate selection bias. ${ }^{12}$ The number of partici- pating hospitals has risen from to 258 sites in 2010 to over 500 in 2014. The inter-rater reliability of data contained within NSQIP databases has been externally validated. ${ }^{13}$

Patients were included if they had a diagnosis of benign cranial nerve neoplasm (International Classification of Disease, Ninth Edition, Clinical Modification (ICD-9-CM) code 225.1), were managed as inpatients, and general anesthesia or monitored anesthesia care were utilized. The ICD-9 code 225.1 includes all benign cranial nerve neoplasms and was used as a proxy for vestibular schwannoma, as it epidemiologically represents the large majority of benign cranial nerve neoplasms. Cases were excluded if the primary code was not a cranial procedure, under presumption that these may have been incorrectly coded. These criteria yielded a total of 726 cases. The quantity of vestibular schwannoma cases within the NSQIP databases reflects the annual increase in participating institutions: 28 cases were included in 2010, while 243 cases were included in the 2014 database.

Cases assigned CPT codes 61305, 61345, 61458, and 61500 to 61530 had a single primary code for craniotomy for tumor. For surgeries with skull base approach and/or resection CPT codes, 61590 to 61598 were considered approach codes, while CPT codes 61601 to 61616 were considered tumor resection codes. Ancillary codes for stereotactic image guidance (61781), use of operating microscope (69990), and others were considered separately.

\section{Results}

Codes Billed for Vestibular Schwannoma Surgery Are Highly Variable

Among 274 cases of vestibular schwannoma resections performed by two independent neurosurgery departments, 96 (35\%) were designated with a single craniotomy for tumor resection code while 178 (65\%) were designated with a specific skull base approach and/or excision codes (-Fig. 1A). Cases with a single code for craniotomy for tumor resection were billed with 61520 (86, 89.6\%), 61526 (6, 6.25\%), or 61518 (4, 4.2\%) most commonly (-Fig. 1B). Among cases designated with specific skull base approach and/or excision codes, 61595 (70, 39.3\%), 61596 (38, 21.3\%), and 61591 (5, 2.8\%) were the most common approach codes, while 61616 (160, 89.9\%), $61606(9,5.1 \%)$, and $61615(3,1.7 \%)$ were the most frequently used resection codes (- Fig. 1C-D). Of note, $21.2 \%$ of cases $(n=58)$ were designated with a skull base excision code without an accompanying approach code, which may reflect the lack of availability of codes submitted by otology for this subset of cases.

The NSQIP database allows coders to enter one primary and multiple secondary codes for each case. In comparison to institutional data, 549 of 726 (75.6\%) vestibular schwannoma resections in the ACS-NSQIP database were assigned a single craniotomy code for tumor approach and resection as the primary code, while 177 (24.4\%) were designated with either a skull base approach or excision primary code (-Fig. 1E). Among cases with a single craniotomy for tumor resection code, 61520 (304, 55.4\%), 61526 (129, 23.5\%), and $61530(63,11.5 \%)$ were the most common (-Fig. 1F). Of 
Table 1 Description of common procedural terminology (CPT) codes used in the institutional and American College Surgeons National Surgical Quality Improvement Program databases for patients who underwent surgery for vestibular schwannoma resections. The most common codes encountered are in bold

\begin{tabular}{|c|c|}
\hline CPT codes & CPT code description \\
\hline \multicolumn{2}{|c|}{ Single approach and excision codes } \\
\hline 61305 & Craniectomy or craniotomy, exploratory; infratentorial (posterior fossa) \\
\hline 61345 & Other cranial decompression, posterior fossa \\
\hline 61458 & Craniectomy, suboccipital; for exploration or decompression of cranial nerves \\
\hline 61500 & Craniectomy; with excision of tumor or other bone lesion of skull \\
\hline 61510 & Craniectomy, trephination, bone flap craniotomy; for excision of brain tumor, supratentorial, except meningioma \\
\hline 61516 & Craniectomy, trephination, bone flap craniotomy; for excision or fenestration of cyst, supratentorial \\
\hline 61518 & $\begin{array}{l}\text { Craniectomy for excision of brain tumor, infratentorial or posterior fossa, except meningioma, cerebellopontine } \\
\text { angle tumor, or midline tumor at base of skull }\end{array}$ \\
\hline 61520 & Craniectomy for excision of cerebellopontine angle tumor \\
\hline 61521 & Craniectomy for excision of midline tumor at base of skull \\
\hline 61526 & Craniectomy, bone flap craniotomy, transtemporal (mastoid) for excision of cerebellopontine angle tumor \\
\hline 61530 & $\begin{array}{l}\text { Craniectomy, bone flap craniotomy, transtemporal (mastoid) for excision of cerebellopontine angle tumor; } \\
\text { combined with middle/posterior fossa craniotomy/craniectomy }\end{array}$ \\
\hline \multicolumn{2}{|c|}{ Approach codes } \\
\hline 61590 & Infratemporal preauricular approach to middle cranial fossa \\
\hline 61591 & Infratemporal postauricular approach to middle cranial fossa \\
\hline 61592 & Orbitocranial zygomatic approach to middle cranial fossa \\
\hline 61595 & Transtemporal approach to posterior cranial fossa \\
\hline 61596 & Transcochlear approach to posterior cranial fossa \\
\hline 61597 & Transcondylar (far lateral) approach to posterior cranial fossa \\
\hline 61598 & Transpetrosal approach to posterior cranial fossa \\
\hline \multicolumn{2}{|c|}{ Excision codes } \\
\hline 61600 & Resection or excision of neoplastic, vascular, or infectious lesion of base of anterior cranial fossa; extradural \\
\hline 61601 & $\begin{array}{l}\text { Resection or excision of neoplastic, vascular, or infectious lesion of base of anterior cranial fossa; intradural, } \\
\text { including dural repair, with or without graft }\end{array}$ \\
\hline 61605 & $\begin{array}{l}\text { Resection or excision of neoplastic, vascular, or infectious lesion of infratemporal fossa, parapharyngeal space, } \\
\text { petrous apex; extradural }\end{array}$ \\
\hline 61606 & $\begin{array}{l}\text { Resection or excision of neoplastic, vascular, or infectious lesion of infratemporal fossa, parapharyngeal space, } \\
\text { petrous apex; intradural, including dural repair, with or without graft }\end{array}$ \\
\hline 61607 & $\begin{array}{l}\text { Resection or excision of neoplastic, vascular, or infectious lesion of parasellar area, cavernous sinus, clivus, or } \\
\text { midline skull base; extradural }\end{array}$ \\
\hline 61608 & $\begin{array}{l}\text { Resection or excision of neoplastic, vascular, or infectious lesion of parasellar area, cavernous sinus, clivus, or } \\
\text { midline skull base; intradural, including dural repair, with or without graft }\end{array}$ \\
\hline 61615 & Resection or excision of neoplastic, vascular or infectious lesion of base of posterior cranial fossa; extradural \\
\hline 61616 & $\begin{array}{l}\text { Resection or excision of neoplastic, vascular, or infectious lesion of base of posterior cranial fossa; intradural, } \\
\text { including dural repair, with or without graft }\end{array}$ \\
\hline
\end{tabular}

these, 34 cases (6.2\%) had an additional secondary approach and/or excision code; 21 of these were coded by the secondary surgical team on the case. Among cases with concurrent skull base approach and excision codes, 61595 (97, 54.8\%), 61596 (24, 13.6\%), and 61591 (16, 9.0\%) were the most common approach codes, while 61616 (106, $59.9 \%), 61615(21,11.9 \%)$, and $61606(15,8.5 \%)$ were the most common excision codes ( $\mathbf{- F i g . ~ 1 G - H ) . ~ O f ~ c a s e s ~ w i t h ~ a ~}$ primary excision or approach code, 27 (15.3\%) did not have a corresponding secondary approach or excision code, including 13 primary approaches and 14 primary excisions.

Given the observed variations in billed codes, we explored possible reasons for such differences within institutional and nationwide data. 


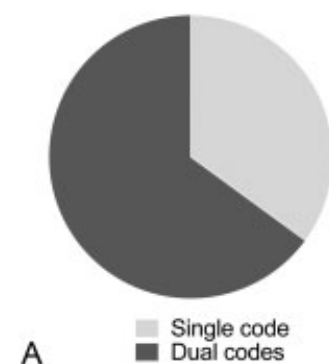

A

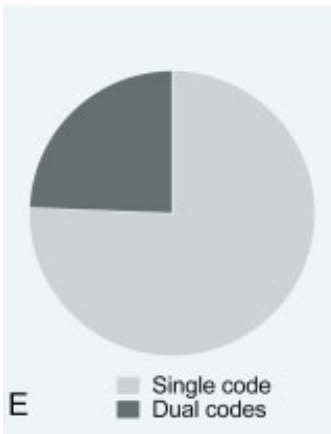

Institutional data
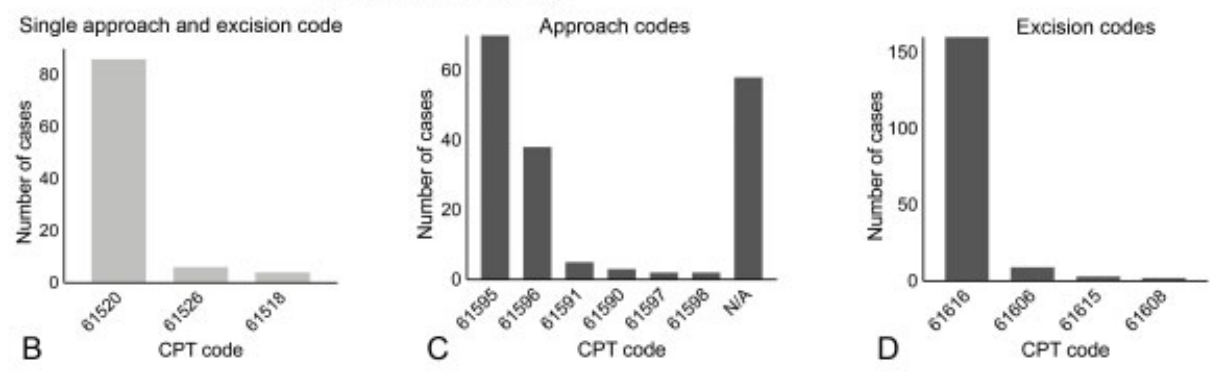

\section{NSQIP data}

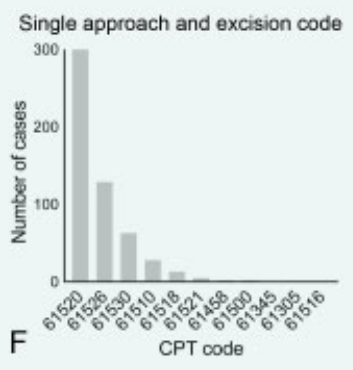

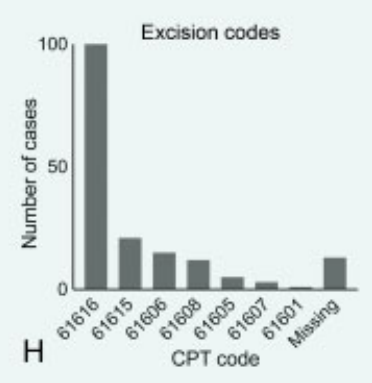

Fig. 1 Incidence of vestibular schwannoma operations with a single primary code or separate approach and resection codes in (A) data from two institutions and (E) National Surgical Quality Improvement Program (NSQIP). Among cases with a single craniotomy for tumor resection codes, distribution of submitted codes at (B) two institutions and (F) within NSQIP. Among cases associated with distinct skull base approach and/or excision codes, distribution of $(C, G)$ approach and $(D, H)$ excision codes encountered at two profiled institutions and NSQIP. Abbreviation: CPT, common procedural terminology.

\section{Codes Billed Do Not Consistently Reflect the Surgical Approach for Vestibular Schwannoma Resection}

We first investigated the association of CPT codes with specific surgical approaches for vestibular schwannoma. Across two institutions, 210 (76.6\%) vestibular schwannoma cases were performed using a retrosigmoid approach, 56 $(20.4 \%)$ by the translabyrinthine approach, and $8(3 \%)$ by the middle fossa approach (- Fig. 2A).

For the retrosigmoid approach, $44.8 \%$ of cases ( $n=94)$ were assigned a single code for craniotomy for tumor resection, with 61520 being the most common (85/94 cases, -Fig. 3B). For cases with distinct skull base approach and/or excision codes $(n=116)$, six approach codes and five excision codes were documented, with the most common being 61595 (66/116 cases) and 61616 (105/116 cases), respectively.

For the translabyrinthine approach, the majority of cases (54/56 cases) were designated with specific skull base approach and/or definitive excision codes, while only $3.6 \%$ (2/56 cases) carried single craniotomy for tumor resection codes. Among these, three approach codes and four excision codes were observed, with the most common being 61596 $(61 \%, 34 / 56)$ for approach and $61616(91 \%, 51 / 56)$ for excision. For the middle fossa approach, one approach code (61591) and three excision codes were encountered.

\section{Patterns of Codes Billed for Vestibular Schwannoma Surgery Vary Across Institution and Surgeon Practices} We compared coding patterns between two independent neurosurgery departments and observed that 61520 was the most common code for cases designated with a single CPT for craniotomy for tumor resection, accounting for $27.5 \%$ (44/160)

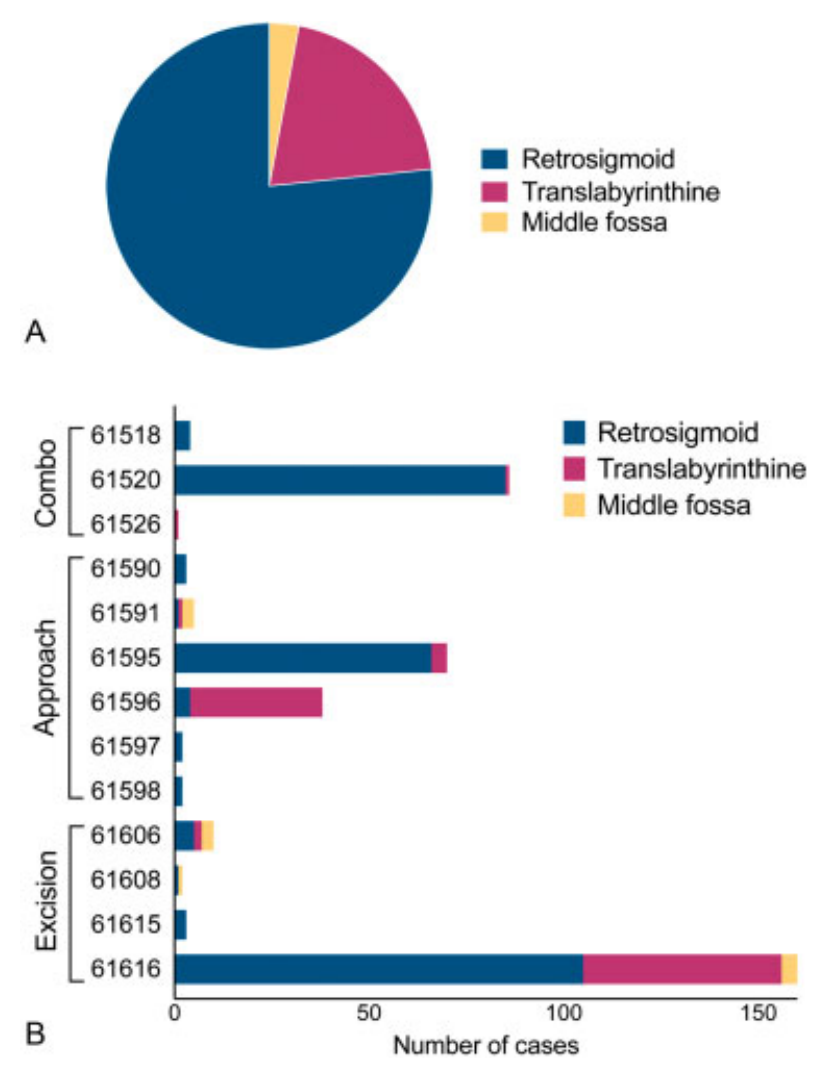

Fig. 2 (A) Incidence of retrosigmoid (76.6\%), translabyrinthine (20.4\%), and middle fossa (3\%) approaches for vestibular schwannoma resection at two academic institutions. (B) Comparison of surgical approach (retrosigmoid, translabyrinthine, and middle fossa) for 274 cases encountered at two neurosurgical practices with their assigned common procedural terminology codes, as sorted by single codes for craniotomy for tumor resection, skull base approach, and definitive excision codes. 
Institutional trend in use of single vs. dual codes

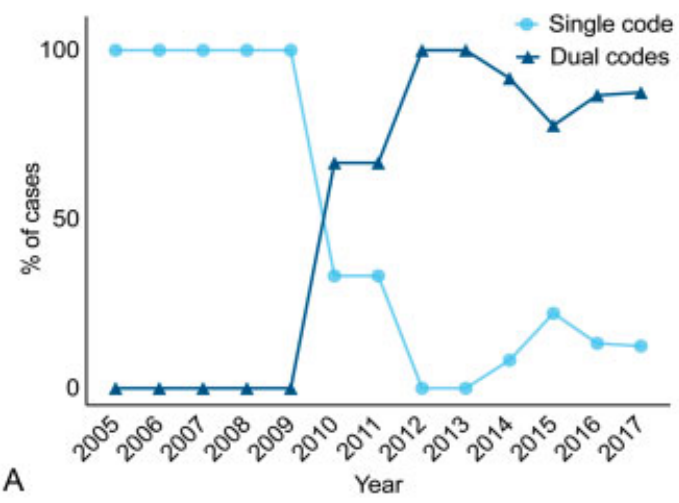

Trend in approach codes in NSQIP

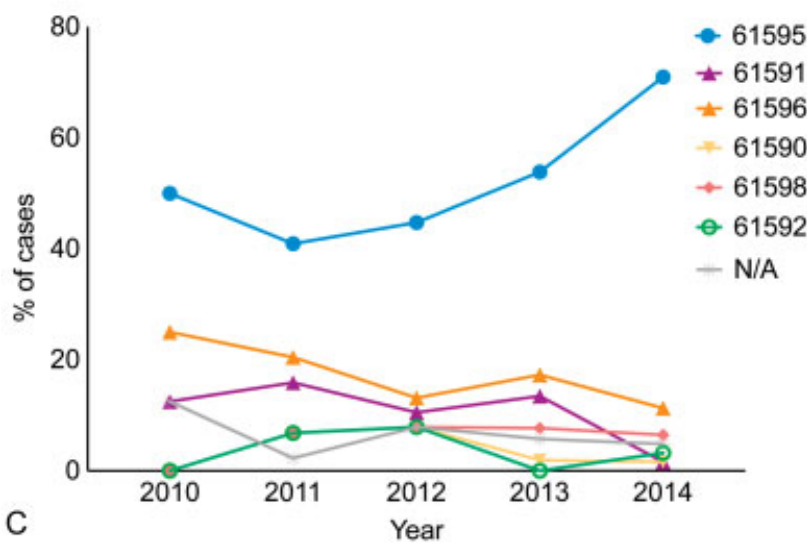

Trend in use of single vs. dual codes in NSQIP

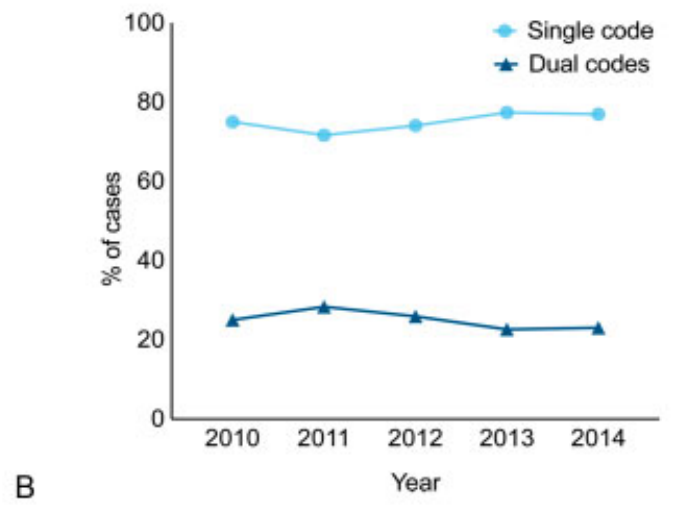

Trend in excision codes in NSQIP

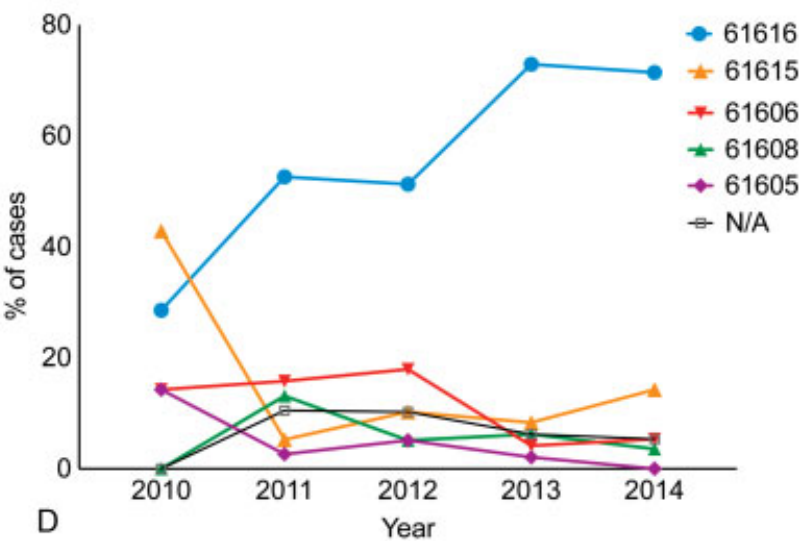

Fig. 3 (A) Skull base-specific approach and excision codes became far more commonly utilized than single craniotomy for tumor resection codes at a single institution from 2005 to 2017. (B) In comparison, the cumulative incidence of single code for craniotomy for tumor resection versus dual skull base codes for approach/resection has remained relatively stable within the National Surgical Quality Improvement Program (NSQIP) database from 2010 to 2014, while specific (C) approach and (D) excision codes have varied significantly in utilization over time.

of one group's cases and 36.8\% (42/114) of the second department's vestibular schwannoma cases. In comparison, over half of cases $(58 / 111,52.3 \%)$ with a specific skull base approach and/or excision code carried only an excision code without an accompanying approach code at one institution, while the other had near concordance $(67 / 68,98.5 \%)$ of both an approach and excision code for dual skull base code cases.

Within the institution with a high frequency of vestibular schwannoma cases with a skull base approach code only, 96.6\% (56/58) of those cases were performed with a neurotology co-surgeon, whose billing reports were not available for analysis. The vast majority $(156 / 160,97.5 \%)$ of cases at this institution were performed by three neurosurgeons over this time period. No clear trends or biases between individual surgeons with respect to code submissions were observed. Within the neurosurgery department with near concordance of dual approach and excision codes for cases designated with skull base specific codes, 40.3\% (27/67) of those cases were performed with a neurotology co-surgeon.

Collectively among the institutional cohort, $71.5 \%$ of surgeries $(n=196)$ were performed by a neurosurgeon and neurotologist team, while $28.5 \%$ of vestibular schwannoma surgeries $(n=78)$ were performed by neurosurgery alone.
Among cases for which CPT codes submitted by neurosurgery and neurotology were both available $(n=68)$, both practices submitted identical primary procedural codes in $58.8 \%$ of cases (most commonly 61520 or 61526 ), using the -62 modifier. In the remaining cases, one practice submitted both an approach code and a definitive code in $90 \%$ of the cases, while the other practice submitted only a definitive excision code (64\% of the time), only an approach code ( $10.7 \%$ of the time), or a paired approach and excision code that differed from the first practice.

\section{Incidence of Skull Base Code Utilization for Vestibular Schwannoma Resection Over Time}

National practice patterns in applying skull base codes for vestibular schwannoma surgery may have changed with increased awareness for coding options. Therefore, we next explored the incidence in use of a single craniotomy for tumor code versus dual skull base approach and excision codes for vestibular schwannoma surgery across time.

We first examined practice trends in a single neurosurgery department with available coding data for vestibular schwannoma resection over a dozen years. At an institutional level, skull base specific approach and excision codes rose in frequency, while single craniotomy for tumor resection 
codes decreased in use ( $\mathbf{- F i g}$. 3A). In comparison, on a national level, the frequency of usage of primary codes representing approach and excision remained relatively stable within the NSQIP databases from 2010 to 2014 (-Fig. 3B). Among single codes for approach and excision, 61520 has remained the most common code used over time. Within skull base approach and excision codes, 61595 and 61616 have remained the most common and have grown in relative incidence (-Fig. 3 C-D).

\section{Discussion}

Nationwide databases are increasingly employed for analyses of trends across the population and may provide a valuable resource for uncommon pathologies such as skull base tumors. $^{2-8}$ Aggregated data mitigate certain biases inherent to single institution data and small sample sizes and has been applied to study risk factors for negative clinical outcomes, readmissions, and re-operations for surgical procedures. Additionally, the availability of CPT codes in large de-identified databases has prompted conclusions regarding specific surgical approaches. We investigated possible limitations of such application of CPT codes for vestibular schwannoma resection.

We observed a spectrum of procedural codes that are associated with vestibular schwannoma resection. Variations in codes may reflect the specific operation performed, surgical practice patterns, and changes in awareness or interpretation of skull base surgery coding options over time. We explored these hypotheses within institutional and nationwide cohorts.

While a few codes represent the majority of the retrosigmoid and translabyrinthine approaches, no surgical approach was exclusively represented by a single code. Conversely, the most common codes were applied to multiple surgical approaches. Therefore, the surgical approach may not be directly inferable from the CPT code and caution should be exercised in using code-based databases to draw specific conclusions related to surgical approach in vestibular schwannoma resection.

CPT codes for skull base procedures not previously represented by existing codes were introduced in 1994. While standard billing codes for vestibular schwannoma resection have been available since before 1994, modified versions of the approaches-collectively referred to as skull base approachesare applied variably from center to center, with different attendant codes. Knowledge and interpretation of these codes remain varied across the United States. Additionally, vestibular schwannoma operations are frequently performed by a team of surgeons, including a neurosurgeon and a neurotologist. Cosurgeons may each submit codes reflective of their contributions to the operation, with the definitive excision code garnering a slightly higher reimbursement than the approach code. Some institutions perform internal splits of payment between surgical teams as well. Furthermore, the choice of billing code confers differences in remuneration and may provide an additional financial variable in practice variation. Taken together, individual practices and agreements between co-surgeons contribute to heterogeneity in coding. This is highlighted by the opposing trends observed in incidence of nonskull base versus skull base approach and excision codes for resection of vestibular schwannoma at a single institution compared with NSQIP.

The institutional data within this study reflect the practice patterns and biases of the select cohort. Likewise, the NSQIP database contains several pertinent limitations. NSQIP coders may not be trained specifically in coding of skull base pathologies. For example, a common excision code observed within the NSQIP data is 61615, which applies to extradural tumor resection and is not relevant to acoustic schwannomas as an intradural tumor. It does not include the specialty of the second attending when one is present, though it does include all CPT codes associated with an anesthesia exposure. It does not include hospital identifiers, preventing analysis of geographic trends or differences between high- and low-volume centers. Cases from earlier years within the NSQIP database may be more susceptible to institutional biases given fewer participating hospitals. The 8-day cycle of data collection and de-identification of hospitals is designed to minimize such selection bias, but limits the quantity of vestibular schwannoma cases included. Furthermore, no specific ICD-9-CM diagnosis code exists for vestibular schwannoma. While vestibular schwannomas represent the most common of benign cranial nerve neoplasms, rarer tumors of other cranial nerves that are encompassed by this diagnosis code may conflate the data as well as vestibular schwannomas that may have been miscoded as an unspecified cerebellopontine angle tumor or a benign tumor of the meninges. Despite these limitations, NSQIP provides a multi-institutional sample of surgical coding and outcomes that may augment single institution resources.

We observed significant variability in coding patterns for vestibular schwannoma resection between institutions and surgical practices, which are not fully reflective of the surgical approach. These results call for discretion in interpretation of data from aggregated billing code-based databases for resection of skull base tumors. Furthermore, guidelines for standardization of CPT assignments for specific surgical approaches may merit review and deepened dissemination in the future.

\section{Conflict of Interest}

None.

\section{Acknowledgments}

The authors express gratitude to Sarah Dizoglio, Jacob Barron, and Cathy Butterworth for their assistance with data acquisition.

\section{References}

1 Karhade AV, Larsen AMG, Cote DJ, Dubois HM, Smith TR. National Databases for Neurosurgical Outcomes Research: options, strengths, and limitations. Neurosurgery 2017. doi: 10.1093/ neuros/nyx408

2 Murphy M, Gilder H, McCutcheon BA, et al. Increased operative time for benign cranial nerve tumor resection correlates with increased morbidity postoperatively. J Neurol Surg B Skull Base 2016;77(04):350-357 
3 Murphy ME, McCutcheon BA, Kerezoudis P, et al. Morbid obesity increases risk of morbidity and reoperation in resection of benign cranial nerve neoplasms. Clin Neurol Neurosurg 2016; 148:105-109

4 Mahboubi H, Haidar YM, Moshtaghi O, et al. Postoperative complications and readmission rates following surgery for cerebellopontine angle schwannomas. Otol Neurotol 2016;37(09): 1423-1427

5 O'Connell BP, Rizk HG, Stevens SM, Nguyen SA, Meyer TA. The relation between obesity and hospital length of stay after elective lateral skull base surgery: an analysis of the American College of Surgeons National Surgical Quality Improvement Program. ORL J Otorhinolaryngol Relat Spec 2015;77(05):294-301

6 Schwam ZG, Michaelides E, Kuo P, Hajek MA, Judson BL, Schutt C. Thirty-day morbidity and mortality following otologic/neurotologic surgery: analysis of the national surgical quality improvement program. Laryngoscope 2018;128(06):1431-1437

7 Vedantam A, Mayer RR, Staggers KA, Harris DA, Pan IW, Lam SK. Thirty-day outcomes for posterior fossa decompression in children with Chiari type 1 malformation from the US NSQIP-Pediatric database. Childs Nerv Syst 2016;32(11):2165-2171
8 Cole T, Veeravagu A, Zhang M, et al. Retrosigmoid versus translabyrinthine approach for acoustic neuroma resection: an assessment of complications and payments in a longitudinal administrative database. Cureus 2015;7(10):e369

9 Rolston JD, Han SJ, Chang EF. Systemic inaccuracies in the National Surgical Quality Improvement Program database: implications for accuracy and validity for neurosurgery outcomes research. J Clin Neurosci 2017;37:44-47

10 Oravec CS, Motiwala $M$, Reed $K$, et al. Big data research in neurosurgery: a critical look at this popular new study design. Neurosurgery 2018;82(05):728-746

11 Bohl DD, Basques BA, Golinvaux NS, Baumgaertner MR, Grauer JN Nationwide Inpatient Sample and National Surgical Quality Improvement Program give different results in hip fracture studies. Clin Orthop Relat Res 2014;472(06):1672-1680

12 Khuri SF. The NSQIP: a new frontier in surgery. Surgery 2005;138 (05):837-843

13 Sellers MM, Merkow RP, Halverson A, et al. Validation of new readmission data in the American College of Surgeons National Surgical Quality Improvement Program. J Am Coll Surg 2013;216 (03):420-427 